\title{
Effects of electron acceptors on removal of antibiotic resistant Escherichia coli, resistance genes and class 1 integrons under anaerobic conditions
}

\author{
Heyang Yuan a , Jennifer H. Miller ${ }^{\mathrm{a}}$, Ibrahim M. Abu-Reesh ${ }^{\mathrm{b}}$, Amy Pruden ${ }^{\mathrm{a}}$, Zhen He ${ }^{\mathrm{a}, *}$ \\ a Department of Civil and Environmental Engineering, Virginia Polytechnic Institute and State University, Blacksburg, Virginia 24061, USA \\ ${ }^{\mathrm{b}}$ Department of Chemical Engineering, Qatar University, P.O. Box 2713, Doha, Qatar
}

\section{H I G H L I G H T S}

- The fate of an antibiotic resistant $E$. coli stain and its ARGs in BES is studied.

- The removal of the E. coli and its ARGs is enhanced with decreased current.

- The ARGs are removed when the host E. coli dies and persist when the host survives.

- The survival of the E. coli depends on the availability of electron acceptors.

\section{A R T I C L E I N F O}

\section{Article history:}

Received 28 May 2016

Received in revised form 28 June 2016

Accepted 1 July 2016

Available online 19 July 2016

Editor: Jay Gan

\section{Keywords:}

Antibiotic resistant bacteria

Antibiotic resistant genes

Bioelectrochemical systems

Electron acceptors

\section{G R A P H I C A L A B S T R A C T}

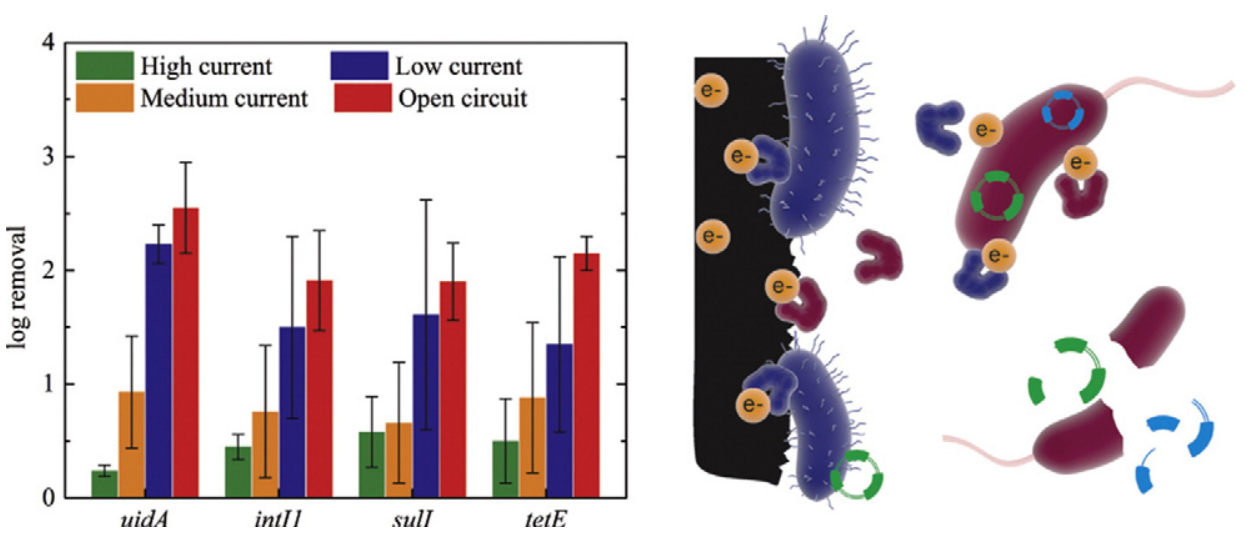

\begin{abstract}
A B S T R A C T
Anaerobic biotechnologies can effectively remove antibiotic resistant bacteria (ARB) and antibiotic resistance genes (ARGs), but there is a need to better understand the mechanisms. Here we employ bioelectrochemical systems (BES) as a platform to investigate the fate of a native tetracycline and sulfonamide-resistant Escherichia coli strain and its ARGs. The E. coli strain carrying intI1, sull and tet(E) was isolated from domestic wastewater and dosed into a tubular BES. The BES was first operated as a microbial fuel cell (MFC), with aeration in the cathode, which resulted in enhanced removal of $E$. coli and ARGs by $\sim 2 \log$ (i.e., order of magnitude) when switched from high current to open circuit operation mode. The BES was then operated as a microbial electrolysis cell (MEC) to exclude the effects of oxygen diffusion, and the removal of $E$. coli and ARGs during the open circuit configuration was again 1-2 $\log$ higher than that at high current mode. Significant correlations of $E$. coli vs. current $\left(\mathrm{R}^{2}=0.73\right)$ and ARGs vs. E. coli ( $\mathrm{R}^{2}$ ranged from 0.54 to 0.87 ), and the fact that the BES substrate contained no electron acceptors, implied that the persistence of the E. coli and its ARGs was determined by the availability of indigenous electron acceptors in the BES, i.e., the anode electrode or the electron shuttles generated by the exoelectrogens. Subsequent experiments with pure-culture tetracycline and sulfonamide-resistant $E$. coli being incubated in a two-chamber MEC and serum bottles demonstrated that the E. coli could survive by respiring anode electrode and/or electron shuttles released by exoelectrogens, and ARGs persisted with their host E. coli.
\end{abstract}

(C) 2016 Elsevier B.V. All rights reserved.

\footnotetext{
* Corresponding author.

E-mail address: zhenhe@vt.edu (Z. He).
} 


\section{Introduction}

Antibiotic resistant bacteria (ARB) and antibiotic resistance genes (ARGs) are emerging environmental contaminants posing serious concern for public health (Pruden et al., 2006). Bacterial pathogens carrying ARGs can survive in the presence of antibiotics through several mechanisms (Wright, 2012), thereby enriching resistant strains and lowering drug efficacy. A major concern is that ARGs can be shared among different species through horizontal gene transfer (Stokes and Gillings, 2011), which appears to be a rare, but important event in the spread of antibiotic resistance (Durso et al., 2012; Forsberg et al., 2014; Stalder et al., 2012). The rise in percentages of resistant bacteria has steadily followed the rise in antibiotic use in humans and agriculture. Both human and livestock excrete antibiotics, antibiotic metabolites, ARBs and ARGs into agricultural waste and domestic wastewater, treatment processes for which are not intentionally designed for their removal, with eventual impact on receiving environments (Hong et al., 2013; Storteboom et al., 2010a; Storteboom et al., 2010b). The activated sludge process in conventional wastewater treatment plants (WWTPs) may create a suitable environment for the proliferation of ARBs and horizontal transfer of ARGs (Moura et al., 2012; Pruden, 2013; Schlüter et al., 2007; Szczepanowski et al., 2009). An elevated level of ARGs has been observed in WWTP effluent after mixed-media filtration and hypochlorite disinfection (LaPara et al., 2011). The New Delhi metallo- $\beta$-lactamase (NDM-1) genetic element, which imparts resistance to a wide variety of antibiotics, has recently been found in WWTP effluent in China, and was able to be propagated to indigenous bacteria in the receiving environment (Luo et al., 2014). Given the environmental impacts and persistence of ARBs and ARGs, there is an urgent need to understand removal mechanisms and take steps towards formulation of effective removal strategies.

The fate of ARBs and ARGs in anaerobic treatment processes, which represent an approach for energy efficient wastewater treatment, is of great interest. It has been reported that a two-stage thermophilic/ mesophilic anaerobic digestion system can efficiently remove ARGs associated with tetracycline resistance (Ghosh et al., 2009). In another study, both the mesophilic and thermophilic anaerobic digesters showed approximately $1 \log$ removal of nine ARGs, while a longer solids retention time and elevated temperature were suspected to play an important role in their fate (Ma et al., 2011). The removal rate and efficiency of ARGs encoding tetracycline resistance could be increased with increased temperature of the digester (Diehl and LaPara, 2010). Anaerobic digestion was also used as pre-treatment for aerobic stirred tank reactors and noticeably enhanced the removal of a variety of ARGs (Christgen et al., 2015). While these prior studies demonstrated that ARBs and ARGs can be reduced under some circumstances by anaerobic digesters, there remains a knowledge gap with respect to the removal mechanisms.

Bioelectrochemical systems (BES), such as microbial fuel cells (MFCs) and microbial electrolysis cells (MECs), are novel anaerobic biotechnologies for water and wastewater treatment with simultaneous energy recovery (Logan and Rabaey, 2012; Lu et al., 2015; Sevda et al., 2015). Electrochemically active microorganisms (i.e., exoelectrogens) consume organics in wastewater and respire by releasing electrons extracellularly, and the anode electrode serves as the main electron acceptor (Lovley, 2012). The fact that oxidation and reduction reactions are separate and controllable makes BES a versatile research platform for various functions, such as the removal of nutrients, organic pollutants and heavy metals (Kelly and He, 2014; Wang and Ren, 2013; Zeng et al., 2015; Zhang and Angelidaki, 2014). However, the fate of ARBs and ARGs in BES has not yet been reported to our knowledge.

The objective of this study was to investigate the fate of a native tetracycline and sulfonamide-resistant $E$. coli strain and its ARGs in BES, and to study the possible effects of electron acceptors on removal. The E. coli strain was isolated from primary WWTP effluent and confirmed to carry the sulfonamide-resistance gene sull, the tetracycline-resistance gene tet(E) and class 1 integrase gene intI1. The E. coli was spiked into a tubular BES anode, which was operated in either an MFC or an MEC mode, and was subject to a range of currents by adjusting the external resistance. Synthetic wastewater with acetate as carbon source and without any electron acceptor was used as the substrate. The removal of the E. coli and the target ARGs were tracked before and after treatment using real-time quantitative polymerase chain reaction (qPCR). To further examine the effects of the electrode and electron shuttles as the electron acceptors on removal, pureculture tetracycline and sulfonamide-resistant $E$. coli were incubated in a two-chamber MEC, or in serum bottles amended with autoclaved effluent from the tubular BES.

\section{Materials and methods}

\subsection{Preparation of antibiotic resistant E. coli}

Primary effluent collected from a local WWTP (Peppers Ferry, VA, USA) was diluted 1:50 in LB broth that was amended with $50 \mathrm{mg} \mathrm{L}^{-1}$ tetracycline and sulfamethoxazole (above the minimum inhibitory concentrations for E. coli) (Andrews, 2001). The selection of pure-culture antibiotic resistant $E$. coli was performed as follow: the broth was incubated aerobically at room temperature overnight and spread on HiCrome $^{\mathrm{TM}} \mathrm{m}$-TEC agar (Sigma-Aldrich, MO, USA); the agar was incubated according to the manufacturer's instruction and a single colony was transferred into antibiotic-amended LB broth. The broth-agar incubation was repeated three times. The obtained pure culture was then incubated aerobically at room temperature with constant stirring, and bacterial growth was monitored by measuring the optical density at $600 \mathrm{~nm}$. After $12 \mathrm{~h}$ of incubation, the growth reached mid-log phase. The culture $(20 \mathrm{~mL}$ ) was collected and washed with $10 \mathrm{mM}$ PBS (phosphate buffer saline). The PBS was discarded after centrifugation and the pellet was ready for use. After the collection step, $1 \mathrm{~mL}$ of the culture was transferred into $24 \mathrm{~mL}$ of fresh antibiotic-amended LB broth for next batch of incubation and the culture was collected every $12 \mathrm{~h}$.

\subsection{BES construction and operation}

A tubular BES was constructed as previously described (Yuan et al., 2015a). Briefly, a cation exchange membrane (CMI-7000, Membrane International, Inc., NJ, USA) tube was made to form the anode chamber $(\sim 250 \mathrm{~mL}$ ) containing a carbon brush (Gordon Brush Mfg. Co., Inc., CA, USA) as the anode electrode. A piece of $300-\mathrm{cm}^{2}$ carbon cloth (Zoltek, MO, USA) was coated with $0.1 \mathrm{mg} \mathrm{cm}^{-2} \mathrm{Pt} / \mathrm{C}$ and wrapped around the cation exchange membrane tube as the cathode electrode. The BES was placed in a tubular PVC container, which served as the cathode chamber $(\sim 1000 \mathrm{~mL})$. The anode and cathode electrodes were connected by titanium wire across a resistance decade box. Synthetic wastewater was used as the anolyte (per liter of deionized water): sodium acetate $1.2 \mathrm{~g} ; \mathrm{NaCl}, 0.5 \mathrm{~g} ; \mathrm{MgSO}_{4}, 0.015 \mathrm{~g} ; \mathrm{CaCl}_{2}, 0.02 \mathrm{~g} ; \mathrm{KH}_{2} \mathrm{PO}_{4}$, $0.265 \mathrm{~g} ; \mathrm{K}_{2} \mathrm{HPO}_{4}, 0.535 \mathrm{~g} ; \mathrm{NaHCO}_{3}, 8.4 \mathrm{~g}$; and trace element, $1 \mathrm{~mL}(\mathrm{He}$ et al., 2006). The catholyte was $100 \mathrm{mM}$ PBS $\left(\mathrm{KH}_{2} \mathrm{PO}_{4}, 5.3 \mathrm{~g} \mathrm{~L}^{-1}\right.$; and $\mathrm{K}_{2} \mathrm{HPO}_{4}, 10.7 \mathrm{~g} \mathrm{~L}^{-1}$ ). A peristaltic pump (BT600-2J, Langer Instruments, NJ, USA) was used to recirculate the electrolyte at a flow rate of $\sim 96 \mathrm{~mL} \mathrm{~min}^{-1}$.

The BES was inoculated with anaerobic sludge and operated under MFC mode by aerating the catholyte at room temperature. After a one month of start-up period, stable current generation was obtained, indicating that the BES was well acclimated. No colonies formed when the BES effluent was spread on $\mathrm{m}$-TEC agar, suggesting that the number of background $E$. coli was negligible. The previously prepared tetracycline and sulfonamide-resistant $E$. coli pellet was then resuspended in 250 $\mathrm{mL}$ of synthetic wastewater and fed in the BES anode. The BES was batch-operated, and the substrate was replaced every $24 \mathrm{~h}$. The anode influent and effluent $(1 \mathrm{~mL})$ were collected and stored at $-80{ }^{\circ} \mathrm{C}$ for DNA analysis. The fate of the E. coli and ARGs was studied for the effect 
of current generation (Table 1). The experiments were conducted at room temperature in triplicate.

After the MFC experiments, the BES was operated under the MEC mode to exclude the effects of oxygen diffusion. The aeration in the cathode was stopped, the cathode chamber was sealed and an external voltage of $0.8 \mathrm{~V}$ was applied (3644A, Circuit Specialists, AZ, USA). The fate of the E. coli and ARGs in the MEC was examined following the same experimental procedures as that under the MFC mode.

\subsection{Tracking the fate of pure-culture antibiotic resistant E. coli}

To study the effects of electron shuttles excreted from exoelectrogens on the survival of the E. coli and its ARGs, the tetracycline and sulfonamide-resistant $E$. coli isolate was anaerobically incubated in synthetic wastewater amended with the BES effluent (which was expected to contain oxidized electron shuttles). To obtain such an effluent, the tubular BES was operated under the MEC mode without dosing antibiotic resistant $E$. coli for two weeks. The colony-forming units of tetracycline and sulfonamide-resistant bacteria decreased to within one log of the baseline level that was measured prior to dosing antibiotic resistant E. coli (Supplementary information, SI Fig. S1). The anode effluent was then collected and filtered through a $0.22-\mu \mathrm{m}$ filter membrane. Synthetic wastewater and $100 \mathrm{~mL}$ of the filtered effluent were autoclaved at $121{ }^{\circ} \mathrm{C}$ for $20 \mathrm{~min}$. Filtered riboflavin solution ( $1 \mathrm{mM}$ ) was used as the electron acceptor in positive control groups. The tetracycline and sulfonamide-resistant $E$. coli pellet was resuspended in $24 \mathrm{~mL}$ of the autoclaved synthetic wastewater and transferred to the autoclaved serum bottles. The culture was amended with either $1 \mathrm{~mL}$ of autoclaved synthetic wastewater (blank), filtered anode effluent, filtered/autoclaved anode effluent, or riboflavin solution (positive control). The serum bottles were sparged with nitrogen gas for $5 \mathrm{~min}$, sealed and incubated with constant stirring. After $23 \mathrm{~h}$ of incubation, stirring was stopped for $1 \mathrm{~h}$ to allow dead cells to settle, and 1-mL sample was collected and stored at $-80^{\circ} \mathrm{C}$ for molecular analysis.

To determine the effects of the anode electrode on the removal of the ARB and it ARGs, the E. coli strain was incubated in the anode of a two-chamber MEC. This MEC was built by sandwiching a cation exchange membrane between two glass bottles (Yuan et al., 2014). The anode electrode was a carbon brush and the cathode electrode was two pieces of $20-\mathrm{cm}^{2}$ carbon cloth coated with $0.5 \mathrm{mg} \mathrm{cm}^{-2} \mathrm{Pt} / \mathrm{C}$. The liquid volume of the anode and cathode chamber was 130 and $140 \mathrm{~mL}$, respectively. The anolyte and catholyte were the same as those of the tubular BES. Before experiments, both the MEC reactor and the electrolyte were autoclaved at $121{ }^{\circ} \mathrm{C}$ for $20 \mathrm{~min}$. The E. coli pellet was resuspended in $140 \mathrm{~mL}$ of anolyte and used to inoculate the MEC anode. An external voltage of $0.8 \mathrm{~V}$ was applied on the MEC across the external resistance of $1 \Omega$. The MEC was operated in a batch mode for $24 \mathrm{~h}$ with constant stirring. An MEC operated under open circuit condition served as the control. After $23 \mathrm{~h}$ of incubation, stirring in the anode was stopped for $1 \mathrm{~h}$ and $1-\mathrm{mL}$ sample was collected and stored

Table 1

Performance of the tubular BES under MFC or MEC mode and different current, DO and pH of the anode effluent. H: high current; M: medium current; L: low current; O: open circuit (zero current). CE: Coulombic efficiency. DO: dissolved oxygen. Standard deviation was calculated with triplicate reactor runs.

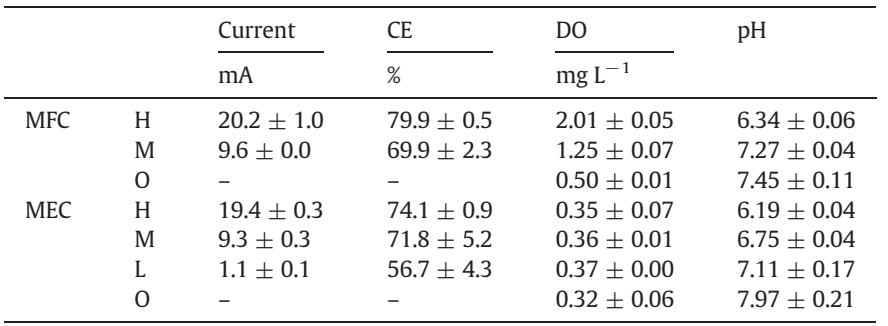

at $-80^{\circ} \mathrm{C}$ for molecular analysis. Both the serum bottle and MEC experiments were conducted at room temperature in triplicate.

\subsection{Molecular analyses}

DNA in the synthetic wastewater before and after treatment was exacted using PureLink ${ }^{\circledR}$ Genomic DNA Mini Kit (Thermo Fisher Scientific, MA, USA). PCR assays were performed with GoTaq ${ }^{\circledR}$ Green Master Mix (Promega, WI, USA) on a thermal cycler (Mastercycler® nexus gradient, Eppendorf, NY, USA). The culture isolated with HiCrome ${ }^{\mathrm{TM}} \mathrm{m}-\mathrm{TEC}$ agar was confirmed to be $E$. coli by detecting the $E$. coli specific gene uidA (Maheux et al., 2009). A series of PCR assays served to detect ARGs (Ma et al., 2011). The presence of sulI, tet(E) and intI1 was confirmed to be of the expected size using gel electrophoresis (SI Fig. S2). PCR was also used to amplify bacterial 16S rRNA genes as an indicator of the total number of bacteria. Details of the primers can be found in SI Table S1.

PCR products of those genes were cloned with TOPA TA Cloning Kit (Invitrogen, CA, USA) and plasmids were isolated using QIAprep Spin Miniprep Kit (Qiagen, CA, USA). Six-point standard curves were constructed from 10-fold serial dilutions of plasmid ranging from $10^{9}$ to $10^{3}$ gene copies $\mu \mathrm{L}^{-1}$. The highest concentration of the plasmid standard for sull was excluded, making it a five-point standard curve to maintain the linear range. Quantitative PCR was performed on a Real Time PCR System (7300, Applied Biosystems, CA, USA) with SsoFast Evagreen Supermix (Bio-Rad Laboratories, CA, USA) to quantify bacterial 16S rRNA gene, uidA, intI1 and sulI, tet(E). The qPCR protocols have been validated and reported in a previous study (Ma et al., 2011). A dilution series was performed and 1:20 was selected and applied across DNA extracts to minimize inhibitory effects. Samples were quantified in triplicate with standards and a negative control included in every run. The average slope of all qPCR assays was $-3.32 \pm 0.00$ with $\mathrm{R}^{2}>0.98$.

\subsection{Measurement and statistics}

The concentration of the plasmid (ng $\mu \mathrm{L}^{-1}$ ) was measured using a spectrophotometer (NanoDrop Lite, Thermo Fisher Scientific, DE, USA) and converted to gene copies $\mu \mathrm{L}^{-1}$ as previously described (Pei et al., 2006). Because the tubular BES was a mixed-culture system, the concentrations of target genes were normalized to that of 16S rRNA gene to minimize the variance in total bacterial population size and to gain insight into the possibility of horizontal gene transfer (Knapp et al., 2008; Liu et al., 2012). The serum bottles and two-chamber MEC contained only pure-culture E. coli, and thus the copy concentrations from those experiments were normalized to the sample volume ( $1 \mathrm{~mL}$ ). Removal of the E. coli and ARGs was calculated as the log difference of the copy concentrations between the influent and effluent. Oneway ANOVA (analysis of variance), two-sample $t$-test and linear correlation with log removal were performed using OriginPro 9. A p-value of $<0.05$ was considered to indicate statistical significance. Current generation was monitored by measuring the voltage on the external resistance using a digital multimeter (2700, Keithley Instruments, $\mathrm{OH}$, USA) at a time interval of $2 \mathrm{~min}$. Soluble COD (chemical oxygen demand) was measured using a colorimeter according to the manufacturer's instruction (DR/890, Hach Company, CO, USA). Coulombic efficiency was calculated according to a previous study (Logan et al., 2006). Dissolved oxygen (DO) was measured using a benchtop DO meter (Orion Star ${ }^{\mathrm{TM}}$ A213, Thermo Fisher Scientific, MA, USA).

\section{Results and discussion}

\subsection{Fate of antibiotic resistant $E$. coli and ARGs under the MFC mode}

Before dosing the tetracycline and sulfonamide-resistant $E$. coli, background $E$. coli in the BES were confirmed to be below detection using $\mathrm{m}$-TEC agar, indicating that indigenous strains were not likely to 
interfere with tracking the spiked strain. The tubular BES was first operated as an MFC with consistent current generation. The current dropped constantly in one cycle due to the consumption of substrate, and rose back to the initial level rapidly after the anolyte was refreshed (Fig. 1), indicating a well-acclimated anode (Logan and Regan, 2006). The average peak current reached $20.2 \mathrm{~mA}$ with a coulombic efficiency of $88.1 \%$ when operated under the high current mode, and decreased to $9.6 \mathrm{~mA}$ when higher external resistance was applied (Table 1). Meanwhile, the DO of the BES anode effluent significantly decreased from $2.01 \mathrm{mg} \mathrm{L}^{-1}$ under the high current condition to $0.50 \mathrm{mg} \mathrm{L}^{-1}$ under the open circuit condition ( $\mathrm{p}<0.05$ ).

Fig. 2 shows that the average removal of the E. coli (according to uidA gene copies) was significantly enhanced from $0.37 \log$ to $2.07 \log$ when the BES operation was switched from high current to open circuit mode ( $t$-test, $\mathrm{p}<0.05$ ). The large standard deviation may be due to the variance of sample collection associated with rapid recirculation

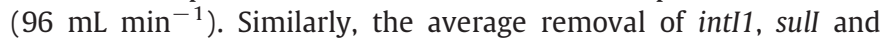
tet(E) was improved by $\sim 2 \log$ when the circuit was disconnected (Fig. 2). The BES anode essentially functioned as an anaerobic digester under the open circuit condition, but the log removal of ARGs achieved (ranging from 2.16 to 3.19 log reduction) here was higher than that reported for mesophilic and thermophilic anaerobic digesters (Ma et al., 2011). This higher removal could be related to the highly specific environment (acetate as the sole carbon source) and limited electron acceptors in the BES anode (no electron acceptors were added in the substrate). E. coli is a facultative organism that can use various chemicals, including nitrate, fumarate and dimethyl sulfoxide as electron acceptors under anaerobic conditions (Unden and Bongaerts, 1997). However, these substances were absent in the synthetic wastewater, making either the oxygen diffused from the cathode or the anode electrode the possible electron acceptor available to the E. coli. The lower removal of ARGs under the condition of high DO together with high current mode was also in agreement with the previous finding that high DO concentration was beneficial for the persistence of tetracycline resistance plasmids in E. coli (Rysz et al., 2013).

\subsection{Fate of antibiotic resistant E. coli and ARGs under MEC mode}

To exclude the possible effects of oxygen diffusion on the removal of the E. coli and ARGs, the BES was operated as an MEC. As a consequence, the DO in the anode effluent was maintained at $0.32-0.37 \mathrm{mg} \mathrm{L}^{-1}$ (Table 1). The current output was adjusted to the same level as the

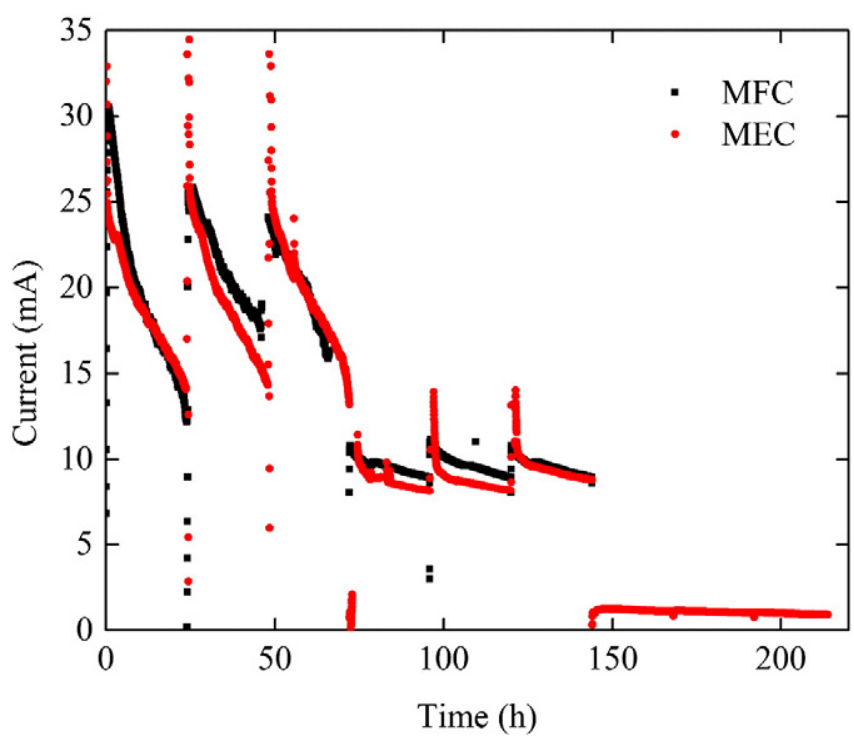

Fig. 1. Current generation of the BES operated under MEC or MFC mode with different external resistance.

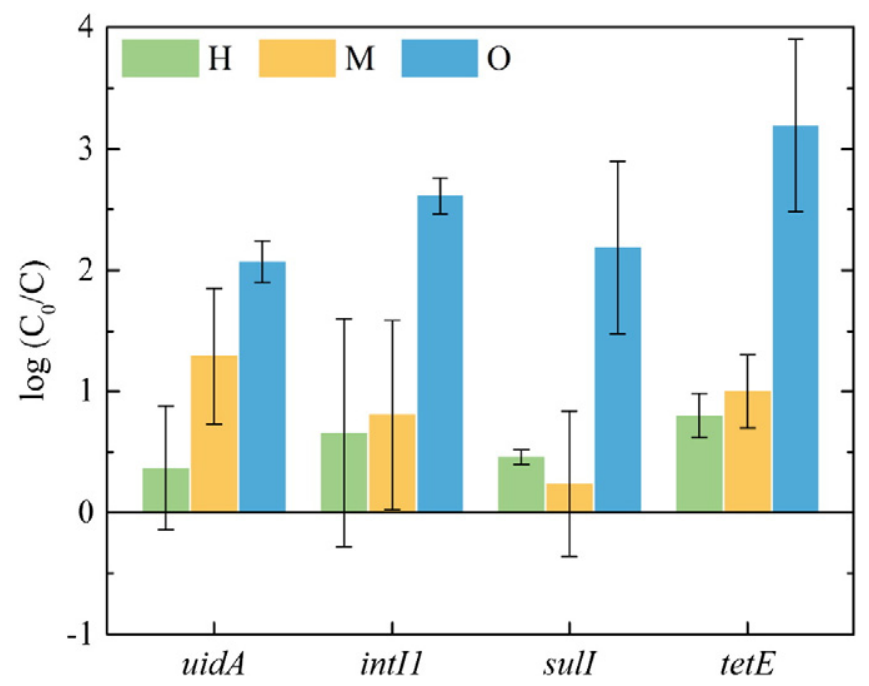

Fig. 2. Removal of tetracycline and sulfonamide-resistant E. coli (uidA), intI1, sulI and tet(E) by the BES operated as an MFC under different current conditions. H: high current; M: medium current; O: open circuit (zero current). Standard deviation was calculated with triplicate reactor runs.

MFC mode through varying external resistance (Fig. 1 and Table 1). The comparable coulombic efficiency suggested that the microbial community did not noticeably change (Kiely et al., 2011; Zhu et al., 2014).

Similar to the results obtained with the MFC mode, more $E$. coli were removed with decreased current (Fig. 3). The reduction of E. coli (uidA) reached $2.23 \log$ under low current conditions, considerably more than the 0.24 log removal observed with high current, and was further enhanced to $2.55 \mathrm{log}$ removal under the open circuit condition. One-way ANOVA supported the conclusion that the removal of E. coli was significantly different across the different current conditions $(p<0.05)$. The removal of ARGs followed the same pattern and was enhanced when the current was decreased. For example, tet(E) was reduced by 1.27 $\log$ more under the open circuit condition relative to the high current condition. The $\mathrm{pH}$ of the anolyte varied as a function of current, but it was maintained within the optimal range for E. coli growth ( $\mathrm{pH}$ 6-8) (Presser et al., 1997), and thus was not likely a significant factor in this study. Therefore, as previously discussed, the availability of electron

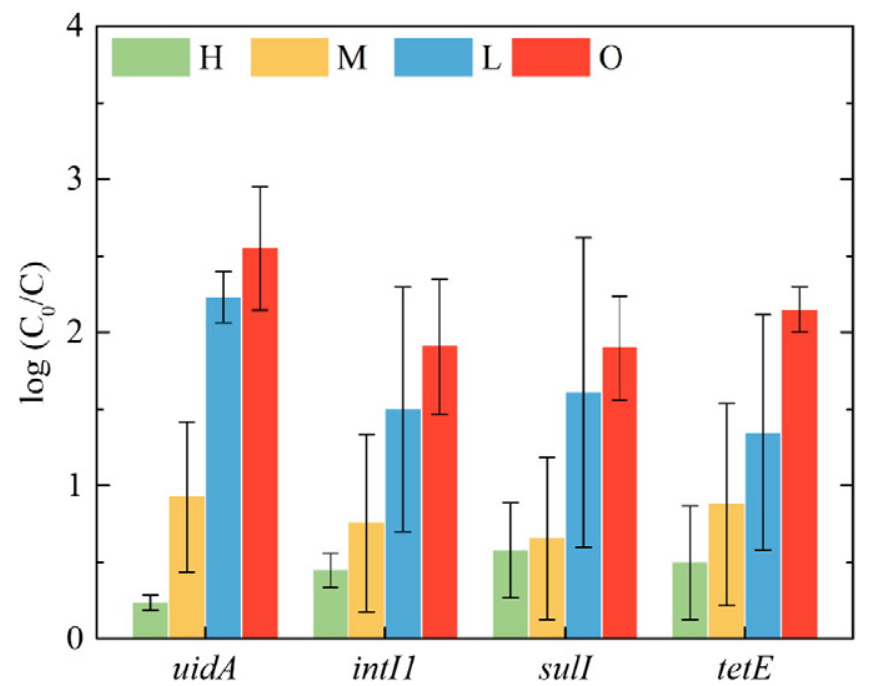

Fig. 3. Removal of tetracycline and sulfonamide-resistant E. coli (uidA), intI1, sulI and tet(E) by the BES operated as an MEC under different current conditions. H: high current; M: medium current; L: low current; O: open circuit (zero current). Standard deviation was calculated with triplicate reactor runs. 
acceptors was likely an important factor accounting for the abundance change of the E. coli and ARGs.

Correlation analyses of $E$. coli vs. current and ARGs vs. E. coli were conducted to further understand potential removal mechanisms. The $\log$ removal of uidA was found to negatively correlate with the current $(\mathrm{p}<0.001)$ (Fig. 4A), implying that current production is beneficial for the survival of antibiotic resistant $E$. coli, likely because they have the opportunity to respire the anode electrode during electricity generation. Previous studies have demonstrated that $E$. coli release electron shuttles to the anode electrode for extracellular electron transfer (Zhang et al., 2006; Zhang et al., 2008). Moreover, prior research indicates that E. coli is likely capable of using electron shuttles released by exoelectrogens (e.g., riboflavin) for respiration (Wang et al., 2015; Wang et al., 2014). Strong correlations between the reduction of ARGs and the reduction of $E$. coli were observed in this study (Fig. 4B-D, $\mathrm{p}<0.05$ ), indicating that the survival of the host $E$. coli is an overarching factor in the persistence of ARGs.

\subsection{Effects of anode electrode}

Based on the aforementioned results, we hypothesized that the survival of antibiotic resistant $E$. coli and the persistence of ARGs could be determined by the availability of an anode electrode as the electron acceptor. To examine this hypothesis, a two-chamber MEC was inoculated with the tetracycline and sulfonamide-resistant $E$. coli. Under closed circuit conditions, the MEC achieved an average current of $0.70 \mathrm{~mA}$ with DO below detection in the anode chamber. The log difference between the copy concentration of uidA in the influent and the effluent was negative (Fig. 5), meaning growth occurred and the abundance of $E$. coli increased by $0.24 \log$. Consistent with the fate of the host, the abundance of intI1, sull and tet(E) increased by $0.28,0.26$ and $0.21 \mathrm{log}$, respectively. In contrast, the abundance of $E$. coli was reduced by $0.13 \mathrm{log}$ under open
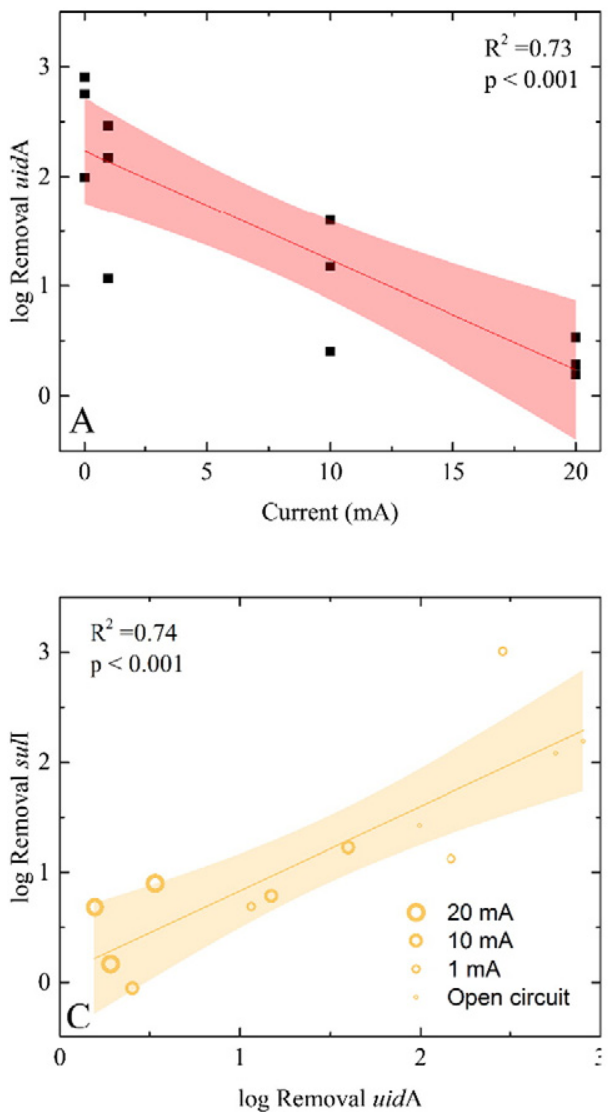

circuit conditions. The removal of ARGs was slightly higher, ranging from 0.23 to $0.25 \log$. It should be noted that the removal of the E. coli and ARGs under open circuit conditions in the two-chamber MEC was noticeably less than that in the tubular at MEC mode ( 2 log, Fig. 3), which could be attributed to the amplification of DNA that persisted from dead cells during qPCR assays (Rudi et al., 2005). The tubular BES contained a mixed-culture that could survive under the open circuit conditions and potentially be transformed by naked DNA from lysed E. coli, whereas DNA accumulated in the pure-culture two-chamber MEC may result in false-positive in qPCR measurement. While the reason of the lower removal in the two-chamber MEC warrants further study, the significant difference in abundance change between the closed and open circuit conditions was in agreement with the proposed removal mechanism.

\subsection{Effects of electron shuttles}

It was also hypothesized that the electron shuttles released by exoelectrogens in the BES anode may serve as electron acceptors after being oxidized by the anode electrode, thereby leading to the survival of antibiotic resistant E. coli and persistence of ARGs. To examine this, the effluent from the tubular BES anode containing oxidized electron shuttles was added in serum bottles to incubate the tetracycline and sulfonamide-resistant E. coli under an anaerobic condition. Similar to the results in the two-chamber MEC, the serum bottles amended with the filtered BES effluent showed negative log difference (i.e., increased abundance) of E. coli and ARGs ranging from -0.12 to $-0.28 \log$ (Fig. $6)$. The abundance increase was slightly higher in the experiments using filtered and autoclaved BES effluent, which did not significantly differ from the results with the filtered effluent ( $p>0.05$ ). Riboflavin as a positive control yielded similar abundance increase as the BES effluent did. On the other hand, the blank group without amendment
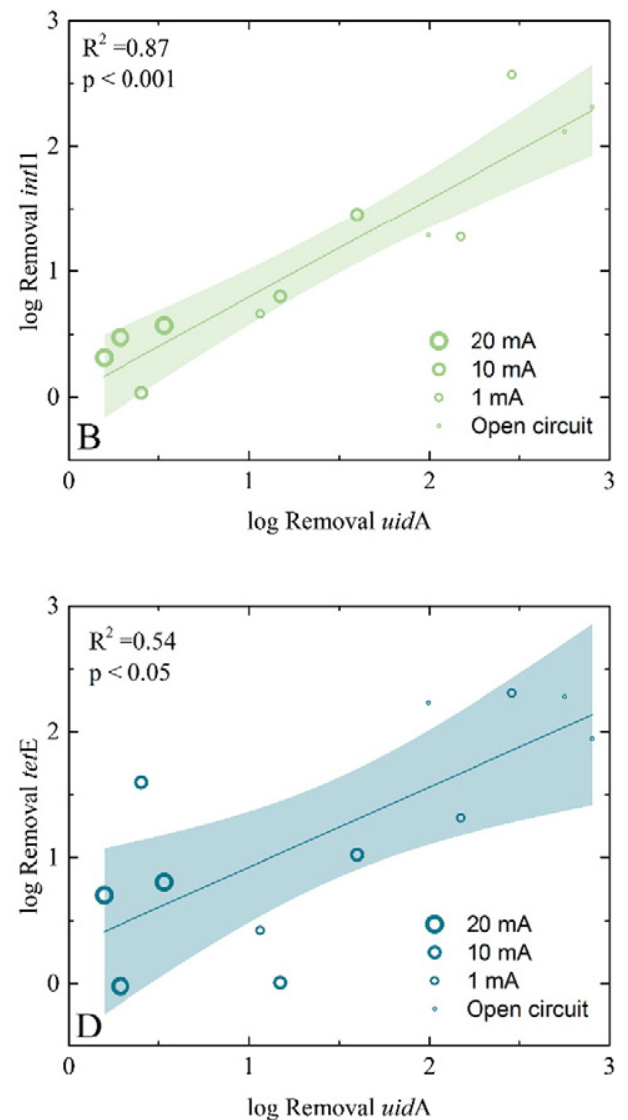

Fig. 4. Linear fitting and Pearson's correlation of (A) uidA vs. current, (B) intI1 vs. uidA, (C) sull vs. uidA and (D) tet(E) vs. uidA. Shadow area represents $95 \%$ confidence interval. 


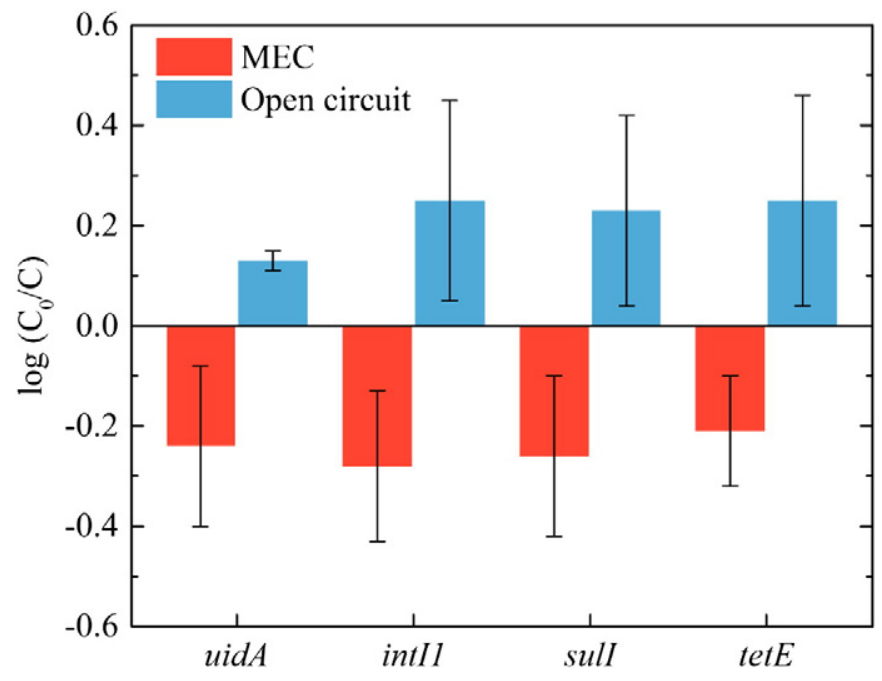

Fig. 5. Abundance change of uidA, intI1, sull and tet(E) in a two-chamber MEC inoculated with pure culture E. coli. Standard deviation was calculated with triplicate reactor runs.

showed reduced E. coli and ARGs by approximately $0.3 \mathrm{log}$, comparable to the results in the two-chamber MEC under the open circuit condition. It has been reported that the addition of riboflavin could enhance electricity generation by E. coli in a pure-culture MFC (Yong et al., 2013). A recent study also revealed the symbiosis of $E$. coli and exoelectrogen Shewanella oneidensis on flavin-mediated extracellular electron transfer (Wang et al., 2015). Thus, the results of this study provide evidence that interspecies interactions related to the utilization of electron acceptors could be playing a critical role in the fate of ARB and ARGs under an anaerobic condition.

\subsection{Perspectives}

The present study, for the first time, used BES as a research platform and $E$. coli as a model organism to gain insight into the removal mechanisms of ARB and ARGs under anaerobic conditions. The removal of exogenously introduced ARBs was significantly enhanced with decreased electrical current $(\mathrm{p}<0.05)$, and ARG removal was significantly correlated with ARB removal $(p<0.05)$. It was therefore hypothesized that ARGs persisted as their host ARB survived, and the survival of ARB was affected by the availability of electron acceptors. Both the anode electrode and the electron shuttles excreted by

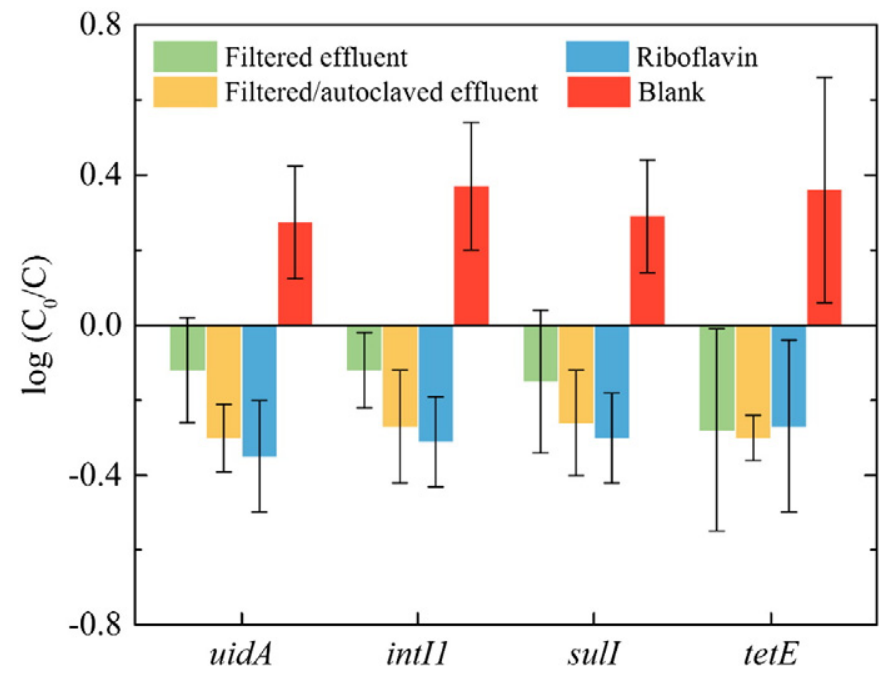

Fig. 6. Abundance change of uidA, int 11 , sull and tetE in serum bottles amended with BES effluent or riboflavin. Standard deviation was calculated with triplicate reactor runs. exoelectrogens were available to ARB for respiration and could have benefitted their survival (Fig. 7A). In the absence of those electron acceptors (i.e., under the open circuit condition), ARB death was likely the main mechanism of ARG removal (Fig. 7B). Further examination of the proposed mechanisms with a two-chamber MEC and serum bottle experiments produced results that agreed well with this explanation. These findings collectively demonstrated that electron acceptor was a key factor affecting the fate of ARBs and ARGs during biological treatment.

ARGs are typically carried on mobile genetic elements, such as plasmids and integrons, which are readily transferred across species through horizontal gene transfer, leading to the persistence of ARGs (Andersson and Hughes, 2011). ARGs are therefore considered the primary contaminants of interest in minimizing the potential to spread antibiotic resistance (Pruden et al., 2006). The persistence and selective increase of ARGs resulting from horizontal gene transfer is a major challenge for biological treatment processes (McKinney et al., 2010; Miller et al., 2016). The slopes in Fig. 4B-D (0.64-0.78) were lower than 1, implying that the removal of ARGs was at a slower rate than that of E. coli. This may be an indication of persistence of extracellular ARGs and/or horizontal gene transfer of ARGs to new hosts. In addition, the slope of sulI vs. E. coli (0.74, Fig. 4C) is distinct from that of tet(E) vs. E. coli (0.64, Fig. 4D). Given the similar lengths of those two amplicons (SI Table S1), the varied slopes suggest that sull and tet(E) decreased at different rates, which is suggestive of gene transfer. While the identification of horizontal gene transfer based on the correlation of ARGs and their host organism is qualitative, quantitative measurement in mixed-cultures is technically difficult. Metagenomics has emerged as a powerful tool to comprehensively understand horizontal gene transfer in environment samples (Fang et al., 2015), and can be used to demonstrate horizontal gene transfer in digesters and BES in future studies.

One of the advantages of BES is energy production. However, the lower removal of E. coli and ARGs under high current operating conditions may present a new challenge if the removal of antibiotic resistance becomes one of the key criteria for wastewater treatment. The enhanced removal at low current suggests that, in order to maintain effluent quality, it may be more suitable to operate BES in high power mode (i.e., the external resistance is adjusted to equal to the internal resistance for achieving the maximum power output) (Jacobson et al., 2011). In addition to the anode treatment, ARBs and ARGs may also be removed in the BES cathode. The anode effluent is commonly used as the catholyte for further COD removal and cathodic reduction reactions. The intermediate products, such as hydroxyl radical and hydrogen peroxide produced by inefficient catalysis of oxygen reduction reactions (Yuan et al., 2015b), may cause oxidative stress of ARBs and damage of ARGs. It has recently been reported that the combination of anaerobic and aerobic treatment remove more ARGs than an anaerobic process alone (Christgen et al., 2015). Moreover, the fate of ARBs and ARGs in other types of BES that focus on biosynthesis or water recovery may differ from that in MFCs and MECs. For example, it will be interesting to explore in a forward osmosis-microbial desalination cell (Yuan et al., 2016) whether ARGs can be transported across the forward osmosis membrane and ion exchange membranes to contaminate the desalinated water.

While new insights were gained into the fate of the antibiotic resistant E. coli and its ARGs in BES, several challenges still remain to be addressed. First, the effects of anode substrate on the fate of ARBs and ARGs remained to be understood. Real wastewater may contain trace amount of antibiotics and exert a selective pressure for ARBs. Second, BES is a unique system that has a stable biofilm with dominant species growing on the electrode. Once exogenous ARGs are horizontally transferred into the exoelectrogens on the electrode, the transformed exoelectrogens may reproduce and slough off the biofilm, thereby releasing ARBs and associated ARGs in the effluent. The interplay of exoelectrogens, ARBs, and horizontal gene transfer of ARGs warrants further studies. 

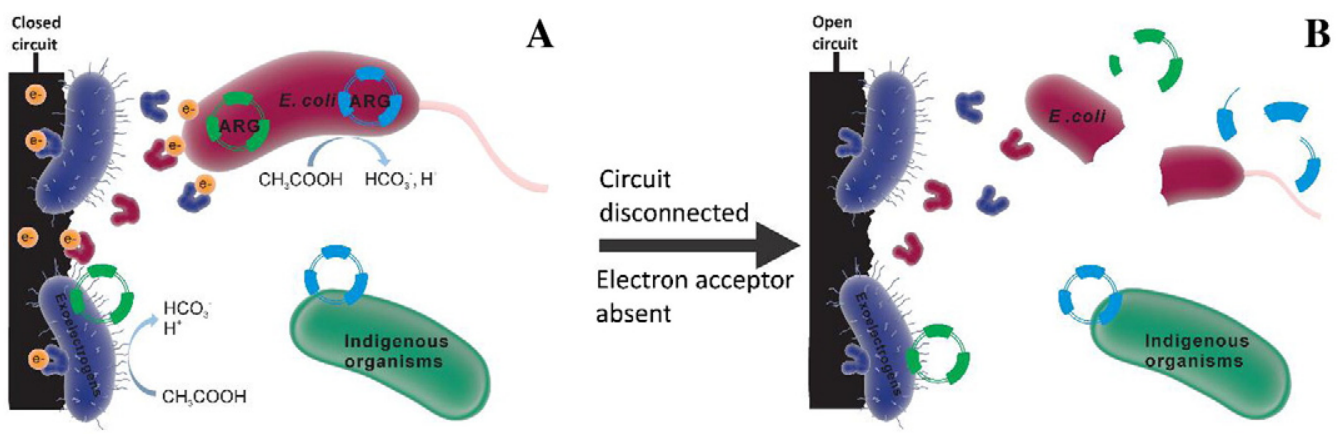

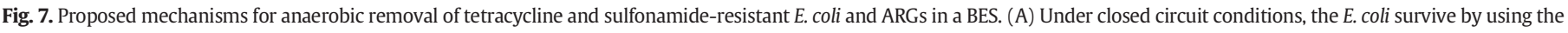

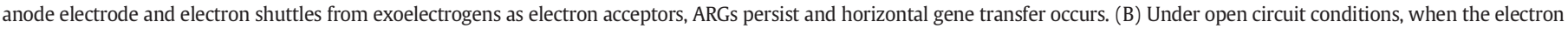
acceptors are absent, the $E$. coli dies and ARGs are removed, horizontal gene transfer may also occur.

\section{Conclusions}

The present study, for the first time, used BES as a research platform to gain insight into the fate of a native tetracycline and sulfonamideresistant $E$. coli strain and its ARGs under anaerobic conditions. When the BES was operated under an MFC mode, the removal of the tetracycline and sulfonamide-resistant E. coli and its ARGs was enhanced with decreased electrical current. The fate of the tetracycline and sulfonamide-resistant $E$. coli and its ARGs in BES operated under an MEC mode was similar to that under the MFC mode. Both the anode electrode and the electron shuttles excreted by exoelectrogens could be used as electron acceptors by the antibiotic resistant $E$. coli for respiration and thus benefit their survival. The ARGs persisted as the E. coli survived, and was removed when the E. coli died.

\section{Acknowledgements}

The authors thank Dr. Husen Zhang at Leidos Biomedical Research, Inc. for the assistance with qPCR. This work was made possible by NPRP grant \# 6-289-2-125 from the Qatar National Research Fund (a member of Qatar Foundation). The statements made herein are solely the responsibility of the authors.

\section{Appendix A. Supplementary data}

Primer information (Table S1), colony-forming units of ARBs in the BES effluent (Fig. S1), gel electrophoresis of ARGs (Fig. S2). Supplementary data associated with this article can be found in the online version, at http://dx.doi.org/10.1016/j.scitotenv.2016.07.002.

\section{References}

Andersson, D.I., Hughes, D., 2011. Persistence of antibiotic resistance in bacterial populations. FEMS Microbiol. Rev. 35, 901-911.

Andrews, J.M., 2001. Determination of minimum inhibitory concentrations. J. Antimicrob. Chemother. 48, 5-16.

Christgen, B., Yang, Y., Ahammad, S.Z., Li, B., Rodriquez, D.C., Zhang, T., et al., 2015. Metagenomics shows that low-energy anaerobic - aerobic treatment reactors reduce antibiotic resistance gene levels from domestic wastewater. Environ. Sci. Technol. 49, 2577-2584.

Diehl, D.L. LaPara, T.M. 2010. Effect of temperature on the fate of genes encoding tetracycline resistance and the integrase of class 1 integrons within anaerobic and aerobic digesters treating municipal wastewater solids. Environ. Sci. Technol. 44, 9128-9133.

Durso, L.M., Miller, D.N., Wienhold, B.J., 2012. Distribution and quantification of antibiotic resistant genes and bacteria across agricultural and non-agricultural metagenomes. PLoS One 7, e48325.

Fang, H., Wang, H., Cai, L., Yu, Y., 2015. Prevalence of antibiotic resistance genes and bacterial pathogens in long-term manured greenhouse soils as revealed by metagenomic survey. Environ. Sci. Technol. 49, 1095-1104.

Forsberg, K.J., Patel, S., Gibson, M.K., Lauber, C.L., Knight, R., Fierer, N., et al., 2014. Bacterial phylogeny structures soil resistomes across habitats. Nature 509, 612-616.

Ghosh, S., Ramsden, S.J., LaPara, T.M., 2009. The role of anaerobic digestion in controlling the release of tetracycline resistance genes and class 1 integrons from municipal wastewater treatment plants. Appl. Microbiol. Biotechnol. 84, 791-796.
He, Z., Wagner, N., Minteer, S.D., Angenent, L.T., 2006. An upflow microbial fuel cell with an interior cathode: assessment of the internal resistance by impedance spectroscopy. Environ. Sci. Technol. 40, 5212-5217.

Hong, P.-Y., Al-Jassim, N., Ansari, M.I., Mackie, R.I., 2013. Environmental and public health implications of water reuse: antibiotics, antibiotic resistant bacteria, and antibiotic resistance genes. Antibiotics 2, 367-399.

Jacobson, K.S., Drew, D.M., He, Z., 2011. Use of a liter-scale microbial desalination cell as a platform to study bioelectrochemical desalination with salt solution or artificial seawater. Environ. Sci. Technol. 45, 4652-4657.

Kelly, P.T., He, Z., 2014. Nutrients removal and recovery in bioelectrochemical systems: a review. Bioresour. Technol. 153, 351-360.

Kiely, P.D., Cusick, R., Call, D.F., Selembo, P.A., Regan, J.M., Logan, B.E., 2011. Anode microbial communities produced by changing from microbial fuel cell to microbial electrolysis cell operation using two different wastewaters. Bioresour. Technol. 102, 388-394.

Knapp, C.W., Engemann, C.A., Hanson, M.L., Keen, P.L., Hall, K.J., Graham, D.W., 2008. Indirect evidence of transposon-mediated selection of antibiotic resistance genes in aquatic systems at low-level oxytetracycline exposures. Environ. Sci. Technol. 42, 5348-5353.

LaPara, T.M., Burch, T.R., McNamara, P.J., Tan, D.T., Yan, M., Eichmiller, J.J., 2011. Tertiarytreated municipal wastewater is a significant point source of antibiotic resistance genes into Duluth-Superior Harbor. Environ. Sci. Technol. 45, 9543-9549.

Liu M, Zhang Y, Yang M, Tian Z, Ren L, Zhang S. Abundance and distribution of tetracycline resistance genes and mobile elements in an oxytetracycline production wastewater treatment system. Environ. Sci. Technol. 2012; 46: 7551-7557.

Logan, B.E., Rabaey, K., 2012. Conversion of wastes into bioelectricity and chemicals by using microbial electrochemical technologies. Science 337, 686-690.

Logan, B.E., Regan, J.M., 2006. Electricity-producing bacterial communities in microbial fuel cells. Trends Microbiol. 14, 512-518.

Logan, B.E., Hamelers, B., Rozendal, R.A., Schroder, U., Keller, J., Freguia, S., et al., 2006. Microbial fuel cells: methodology and technology. Environ. Sci. Technol. 40, 5181-5192.

Lovley, D.R., 2012. Electromicrobiology. Annu. Rev. Microbiol. 66, 391-409.

Lu, Y., Qin, M., Yuan, H., Abu-Reesh, I.M., He, Z., 2015. When bioelectrochemical systems meet forward osmosis: accomplishing wastewater treatment and reuse through synergy. Water 7, 38-50.

Luo, Y., Yang, F., Mathieu, J., Mao, D., Wang, Q., Alvarez, P.J.J., 2014. Proliferation of multidrug-resistant New Delhi metallo- $\beta$-lactamase genes in municipal wastewater treatment plants in northern China. Environ. Sci. Technol. Lett. 1, 26-30.

Ma, Y., Wilson, C.A., Novak, J.T., Riffat, R., Aynur, S., Murthy, S., et al., 2011. Effect of various sludge digestion conditions on sulfonamide, macrolide, and tetracycline resistance genes and class I integrons. Environ. Sci. Technol. 45, 7855-7861.

Maheux, A.F., Picard, F.J., Boissinot, M., Bissonnette, L., Paradis, S., MG, B., 2009. Analytical comparison of nine PCR primer sets designed to detect the presence of Escherichia coli/Shigella in water samples. Water Res. 43, 3019-3028.

McKinney, C.W., Loftin, K.A., Meyer, M.T., Davis, J.G., Pruden, A., 2010. Tet and sul antibiotic resistance genes in livestock lagoons of various operation type, configuration, and antibiotic occurrence. Environ. Sci. Technol. 44, 6102-6109.

Miller, J.H., Novak, JT. Knocke, W.R., Pruden, A., 2016. Antibiotic resistant bacteria survival and horizontal gene transfer control antibiotic resistance gene content of anaerobic digesters. Front. Microbiol. 7.

Moura, A., Oliveira, C., Henriques, I., Smalla, K., Correia, A., 2012. Broad diversity of conjugative plasmids in integron-carrying bacteria from wastewater environments. FEMS Microbiol. Lett. 330, 157-164.

Pei, R., Kim, S.-C., Carlson, K.H., Pruden, A., 2006. Effect of river landscape on the sediment concentrations of antibiotics and corresponding antibiotic resistance genes (ARG). Water Res. 40, 2427-2435.

Presser, K.A., Ratkowsky, D.A., Ross, T., 1997. Modelling the growth rate of Escherichia coli as a function of $\mathrm{pH}$ and lactic acid concentration. Appl. Environ. Microbiol. 63, 2355-2360.

Pruden, A., 2013. Balancing water sustainability and public health goals in the face of growing concerns about antibiotic resistance. Environ. Sci. Technol. 48, 5-14.

Pruden, A., Pei, R., Storteboom, H., Carlson, K.H., 2006. Antibiotic resistance genes as emerging contaminants: studies in northern Colorado†. Environ. Sci. Technol. 40, 7445-7450. 
Rudi, K., Moen, B., Drømtorp, S.M., Holck, A.L., 2005. Use of ethidium monoazide and PCR in combination for quantification of viable and dead cells in complex samples. Appl. Environ. Microbiol. 71, 1018-1024.

Rysz, M., Mansfield, W.R., Fortner, J.D., Alvarez, P.J., 2013. Tetracycline resistance gene maintenance under varying bacterial growth rate, substrate and oxygen availability, and tetracycline concentration. Environ. Sci. Technol. 47, 6995-7001.

Schlüter, A., Szczepanowski, R., Pühler, A., Top, E.M., 2007. Genomics of IncP-1 antibiotic resistance plasmids isolated from wastewater treatment plants provides evidence for a widely accessible drug resistance gene pool. FEMS Microbiol. Rev. 31, 449-477.

Sevda, S., Yuan, H., He, Z., Abu-Reesh, I.M., 2015. Microbial desalination cells as a versatile technology: functions, optimization and prospective. Desalination 371, 9-17.

Stalder, T., Barraud, O., Casellas, M., Dagot, C., Ploy, M.-C., 2012. Integron involvement in environmental spread of antibiotic resistance. Front. Microbiol. 3.

Stokes, H.W., Gillings, M.R., 2011. Gene flow, mobile genetic elements and the recruitment of antibiotic resistance genes into Gram-negative pathogens. FEMS Microbiol. Rev. 35, 790-819.

Storteboom, H., Arabi, M., Davis, J.G., Crimi, B., Pruden, A., 2010a. Identification of antibiotic-resistance-gene molecular signatures suitable as tracers of Pristine River, urban, and agricultural sources. Environ. Sci. Technol. 44, 1947-1953.

Storteboom, H., Arabi, M., Davis, J.G., Crimi, B., Pruden, A., 2010b. Tracking antibiotic resistance genes in the South Platte River basin using molecular signatures of urban, agricultural, and pristine sources. Environ. Sci. Technol. 44, 7397-7404.

Szczepanowski, R., Linke, B., Krahn, I., Gartemann, K.-H., Guetzkow, T., Eichler, W., et al., 2009. Detection of 140 clinically relevant antibiotic-resistance genes in the plasmid metagenome of wastewater treatment plant bacteria showing reduced susceptibility to selected antibiotics. Microbiology 155, 2306-2319.

Unden, G., Bongaerts, J., 1997. Alternative respiratory pathways of Escherichia coli: energetics and transcriptional regulation in response to electron acceptors. Biochim. Biophys. Acta Bioenerg. 1320, 217-234.

Wang, H., Ren, Z.J., 2013. A comprehensive review of microbial electrochemical systems as a platform technology. Biotechnol. Adv. 31, 1796-1807.

Wang, V.B., Yam, J.K.H., Chua, S.-L., Zhang, Q., Cao, B., Chye, J.L.S., et al., 2014. Synergistic microbial consortium for bioenergy generation from complex natural energy sources. Sci. World J. 2014.
Wang, V.B., Sivakumar, K., Yang, L., Zhang, Q., Kjelleberg, S., Loo, S.C.J., et al., 2015. Metabolite-enabled mutualistic interaction between Shewanella oneidensis and Escherichia coli in a co-culture using an electrode as electron acceptor. Sci. Report. 5

Wright, G.D., 2012. Antibiotic resistome: A framework linking the clinic and the environment. John Wiley \& Sons, Inc, Hoboken, New Jersey.

Yong, Y.-C., Yu, Y.-Y., Yang, Y., Liu, J., Wang, J.-Y., Song, H., 2013. Enhancement of extracellular electron transfer and bioelectricity output by synthetic porin. Biotechnol Bioeng. 110, 408-416.

Yuan, H., Li, J., Yuan, C., He, Z., 2014. Facile synthesis of MoS2@CNT as an effective catalyst for hydrogen production in microbial electrolysis cells. ChemElectroChem 1 1828-1833.

Yuan, H., Abu-Reesh, I.M., He, Z., 2015a. Enhancing desalination and wastewater treatment by coupling microbial desalination cells with forward osmosis. Chem. Eng. J. 270, 437-443.

Yuan, H., Hou, Y., Wen, Z., Guo, X., Chen, J., He, Z., 2015b. Porous carbon nanosheets codoped with nitrogen and sulfur for oxygen reduction reaction in microbial fuel cells. ACS Appl. Mater. Interfaces 7, 18672-18678.

Yuan, H., Abu-Reesh, I.M., He, Z., 2016. Mathematical modeling assisted investigation of forward osmosis as pretreatment for microbial desalination cells to achieve continuous water desalination and wastewater treatment. J. Membr. Sci. 502, 116-123.

Zeng, X., Borole, A.P., Pavlostathis, S.G., 2015. Biotransformation of furanic and phenolic compounds with hydrogen gas production in a microbial electrolysis cell. Environ. Sci. Technol. 49, 13667-13675.

Zhang, Y., Angelidaki, I., 2014. Microbial electrolysis cells turning to be versatile technology: recent advances and future challenges. Water Res. 56, 11-25.

Zhang, T., Cui, C., Chen, S., Ai, X., Yang, H., Shen, P., et al., 2006. A novel mediatorless microbial fuel cell based on direct biocatalysis of Escherichia coli. Chem. Commun. 21, 2257-2259.

Zhang, T., Cui, C., Chen, S., Yang, H., Shen, P., 2008. The direct electrocatalysis of Escherichia coli through electroactivated excretion in microbial fuel cell. Electrochem. Commun. 10, 293-297.

Zhu, X., Yates, M.D., Hatzell, M.C., Ananda Rao, H., Saikaly, P.E., Logan, B.E., 2014. Microbia community composition is unaffected by anode potential. Environ. Sci. Technol. 48 1352-1358. 